\title{
Atrioventricular Nodal Function in the Immature Canine Heart ${ }^{1}$
}

\author{
JORGE MCCORMACK, HENRY GELBAND, HUI XU, JUAN VILLAFANE, ADRIENNE STOLFI, \\ AND ARTHUR S. PICKOFF \\ Division of Pediatric Cardiology, Department of Pediatrics, University of Miami School of Medicine, Miami, \\ Florida; the Division of Pediatric Cardiology, Department of Pediatrics, University of Louisville School of \\ Medicine, Louisville, Kentucky [J.V.] and the Division of Pediatric Cardiology, Department of Pediatrics, Tulane \\ University School of Medicine, New Orleans, Louisiana [A.S.P.]
}

\begin{abstract}
Previous studies have suggested that the atrioventricular nodal functional refractory period in the neonate is equal to or shorter than that of the ventricle, providing little or no protection to the ventricle against rapid atrial rates and allowing closely coupled atrial beats to fall within the ventricular vulnerable period. We evaluated atrioventricular node function in 21 mongrel neonatal puppies, 3-15 days old, and 15 adult dogs utilizing intracardiac His bundle recording and stimulation techniques. The mean atrioventricular nodal functional refractory period $(173.1 \pm 20.0 \mathrm{~ms})$ exceeded both the ventricular effective refractory period $(139.5 \pm 14.3)$ and ventricular functional refractory period $(163.3 \pm 14.5)$ in the neonates. Furthermore, the atrioventricular node was the site of limiting antegrade conduction in all neonates. No ventricular arrhythmias were induced by atrial extrastimulation in any of the neonates. The site of conduction delay during atrial extrastimulation was confined to the atrioventricular node in $15 / 16$ neonates $(94 \%)$ while $1 / 16(6 \%)$ had combined nodal and infranodal delay. The neonates developed Wenckebach, at significantly faster heart rates than the adults, but both groups developed Wenckebach at approximately twice the resting heart rate. Retrograde conduction was a consistent finding in the neonates. However, antegrade Wenckebach occurred at a significantly faster heart rate than retrograde Wenckebach suggesting different functional properties. Our data suggest that in the neonatal canine, the atrioventricular node functional refractory period is longer than both the ventricular effective refractory period and ventricular functional refractory period. Furthermore, the degree of protection offered by the neonatal atrioventricular node to the ventricle appears to be comparable to that of the adult. (Pediatr Res 23: 99-103, 1988)
\end{abstract}

\section{Abbreviations}

AV, atrioventricular

AVN, atrioventricular node

VFRP, ventricular functional refractory period

AVNFRP, atrioventricular node functional refractory period

Received August 11, 1986; accepted July 14, 1987.

Correspondence and reprint requests Jorge McCormack, M.D., Pediatric Cardiology, Department of Pediatrics, University of Miami, School of Medicine. P.O. Box 016960, Miami, FL 33101.

Supported in part by the NHLBI National Research Service Award HL07103 (J.M.). NHLBI, Bethesda, MD. Clinical Investigator Award HL00881 (A.S.P.). NHLBI Research Training Grant HL07436, and a grant from the American Heart Association of Greater Miami, Miami, FL.

'Presented in part at the Meeting of the American College of Cardiology, Atlanta, GA, March 10, 1986
PCL, paced cycle length

BCL, basic cycle length

AERP, atrial effective refractory period

AFRP, atrial functional refractory period

AVNERP, atrioventricular node effective refractory period

VERP, ventricular effective refractory period

$P A, P$ wave to low right atrium interval

$\mathrm{HV}$, His to ventricle interval

WHR, Wenckebach heart rate

BHR, basic heart rate

$\mathrm{AH}$, atium to His interval

Previous studies concerning the neonatal AV conduction system have concluded that in the neonate, the AVN is not an effective "filtering device," i.e. that little or no protection is afforded to the neonatal ventricle against premature atrial beats and rapid supraventricular rates by the AVN (1-4). These conclusions are based on observations that 1) the VFRP is longer than the AVNFRP in the immature heart and 2) that ventricular arrhythmias can frequently be induced by supraventricular pacing in the immature heart.

In this study we report AV nodal conduction characteristics in the newborn puppy. Evidence is presented which suggests that, in fact, in the neonatal puppy the AVN does function as an adequate "filtering device" by virtue of having longer refractory periods than the distal conduction system and by offering a comparable degree of protection against rapid supraventricular rates as in the adult.

\section{METHODS}

Two groups of dogs were studied: group I consisted of 21 mongrel puppies, ages 3-15 days (weight 500-1000 g), group II consisted of 15 adult mongrel dogs. All animals were anesthetized with sodium pentobarbital, $30 \mathrm{mg} / \mathrm{kg}$ initial dose, given intraperitoneally in group I and intravenously in group II. Because of the short duration of the experiment (approximately $2 \mathrm{~h}$ ), supplementary sodium pentobarbital was rarely needed. Those animals who required supplementation (i.e. increase in heart rate or blood pressure or presence of spontaneous movements) received one additional dose of sodium pentobarbital $(15 \mathrm{mg} / \mathrm{kg})$ intravenously.

Both groups were intubated and mechanically ventilated. During the course of studies, arterial blood gases are monitored to assure an adequate oxygenation and acid-base status.

Surface lead II EKG and femoral artery blood pressures were constantly monitored. This model has been utilized in our lab- 
oratory for the past $4 \mathrm{yr}$ and significant changes in blood pressure or heart rate rarely occur over a 6 -h period (5).

In group I, a $4 \mathrm{~F}$ quadripolar catheter was inserted through the right femoral vein and placed in the high right atrium and a $5 \mathrm{~F}$ quadripolar catheter was inserted through the right jugular vein and positioned in the RV apex under fluoroscopic guidance. In group II, two $5 \mathrm{~F}$ quadripolar catheters were inserted through the right femoral vein and placed in the high right atrium and right ventricular apex. In both groups, through a left carotid arteriotomy, a $4 \mathrm{~F}$ tripolar catheter was inserted and positioned in the noncoronary cusp of the aorta under fluoroscopic guidance to record the His bundle electrogram.

Intracardiac electrograms were amplified at a filter setting of 40-500 Hz (Electronics for Medicine, DR-6) and recorded on direct writing paper (Kodak Linagraph) at a paper speed of 100 $\mathrm{mm} / \mathrm{s}$.

Programed electrical stimulation was delivered either to the atrium or ventricle at twice diastolic threshold and delivered by a programable digital stimulator (Medtronics, model 5325). Atrial and ventricular extrastimuli were coupled to the eighth paced beat until atrial or ventricular refractoriness, respectively, was reached. Both atrium and ventricle were paced at identical cycle lengths. In this study, we attempted to measure atrial, AV nodal and ventricular refractoriness under basal conditions. However, because of naturally occurring sinus arrhythmia in dogs, the PCL utilized was the longest cycle length which allowed capture of all of the eight paced beats preceding the extrastimulus. Thus, each animal was paced at a different cycle length but only one PCL was used in each animal which was approximately the animal's sinus rate. Because the neonatal BCL is shorter than that of the adult (see "Results"), the neonates as a group were paced at a shorter PCL, than the adults. In group I the PCL ranged from 250 to $320 \mathrm{~ms}$ (mean $=285 \pm 24.0 \mathrm{~ms}$ ) and in group II the PCL ranged from 320 to $450 \mathrm{~ms}$ (mean $=375 \pm$ $42.7 \mathrm{~ms}$ ). In group I, the PCL chosen for each animal ranged from 73 to $93 \%$ of their BCL (mean $=84.2 \pm 5.1 \%$ ) and in group II, the PCL used ranged from 69 to $97 \%$ of the BCL (mean $=84.2 \pm 9.6 \%$ ). While the PCL may affect refractoriness (a shorter PCL results in shorter refractoriness), the results obtained by the selection of the PCL in the above fashion (PCL slightly shorter than BCL), results in the refractoriness of the different cardiac tissues approximating basal conditions (i.e. sinus rate).

Rapid atrial pacing was performed for $30 \mathrm{~s}$ at $25 \mathrm{bpm}$ increments until Wenckebach conduction was observed. In those animals in which retrograde conduction was present, rapid ventricular pacing was performed at $25 \mathrm{bpm}$ increments until retrograde Wenckebach was observed.

Definitions. $\mathrm{S}_{1}, \mathrm{~A}_{1}, \mathrm{H}_{1}, \mathrm{~V}_{1}$ refer to pacing spike artifact, atrial, His bundle, and ventricular electrograms of the basic paced rhythm, while $S_{2}, A_{2}, H_{2}, V_{2}$ refer to the corresponding events recorded with the extrastimulus. AERP: the longest $S_{1} S_{2}$ that fails to result in an atrial depolarization. AFRP: the shortest $A_{1} A_{2}$ in response to any $S_{1} S_{2}$. AVNERP: the longest $A_{1} A_{2}$ measured in the His bundle electrogram that fails to produce an $\mathrm{H}_{2}$. AVNFRP: shortest $\mathrm{H}_{1} \mathrm{H}_{2}$ in response to any $\mathrm{A}_{1} \mathrm{~A}_{2}$. VERP: the longest $S_{1} S_{2}$ that fails to produce a $V_{2}$ upon ventricular pacing. VFRP: the shortest $V_{1} V_{2}$ in response to any $S_{1} S_{2}$.

Statistical analyses comparing observations in the neonatal and adult groups were performed using a two tailed $t$ test. A $p$ value less than 0.05 was considered significant.

\section{RESULTS}

Resting intervals (Table 1). The BCL was significantly shorter in the neonates (339.9 $\pm 34.1 \mathrm{~ms}$ group I; $449.5 \pm 64.6$, group II, $p<0.001)$ and correspondingly to BHR was significantly faster (178.2 \pm 18.9 in group I; $136.1 \pm 19.8$ group II, $p<0.001)$.

Small but significant increases with age were seen in the PA interval $(7.0 \pm 5.0 \mathrm{~ms}$ group I; $12.7 \pm 9.8 \mathrm{~ms}$ group II, $p<0.05)$ and $\mathrm{HV}$ intervals $(17.8 \pm 3.1 \mathrm{~ms}$ group $\mathrm{I} ; 24.5 \pm 7.8 \mathrm{~ms}$ group II, $p<0.05$ ).

The AH interval, a measure of AV nodal conduction time, although slightly longer in the adult, was not significantly different from the neonate $(56.8 \pm 6.9 \mathrm{~ms}$ group I; $60.5 \pm 12.3$ group II, NS).

Atrial and ventricular refractory periods (Table 2). Both the atrial effective and functional refractory periods were shorter in the neonate than in the adult (AERP: $59.0 \pm 11.4$ ms group I; $136.3 \pm 19.7$ group II, $p<0.001 /$ AFRP: $97.4 \pm 15.5 \mathrm{~ms}$ group I: $176.9 \pm 18.5 \mathrm{~ms}$ group II, $p<0.001)$. The ventricular effective and functional refractory periods were also shorter in the neonate (VERP: $139.5 \pm 14.3 \mathrm{~ms}$ group I; $163.0 \pm 15.8 \mathrm{~ms}$ group II, $p<$ 0.001 /VFRP: $163.3 \pm 14.5 \mathrm{~ms}$ group I; $193.3 \pm 21.3 \mathrm{~ms}$ group II, $p<0.001$ ). The differences between the neonatal and adult atrial refractory periods were greater than the differences in the ventricular refractory periods. These findings are consistent with previous observations (5).

$A V$ nodal function. AV Nodal Refractoriness and $A V$ Conduction Characteristics (Table 2). As a measure of the response of the AV node to supranodal input, the AVNERP was determined in both age groups. The AVNERP could be accurately measured in 16 of 21 neonates while in five neonates, atrial refractoriness was reached before the AVNERP could be determined. In 11 of the 15 adult dogs, the AV conduction was limited by atrial refractoriness. Of those neonates in whom the AVNERP was

Table 2. Refractory periods

\begin{tabular}{lcc}
\hline $\begin{array}{c}\text { Parameter } \\
(\text { mean } \pm \mathrm{SD})\end{array}$ & Neonates & Adults \\
\hline & $(n=21)$ & $(n=15)$ \\
AERP & $59.0 \pm 11.4$ & $136.3 \pm 19.7$ \\
AFRP & $97.4 \pm 15.5$ & $176.9 \pm 18.5$ \\
AVNERP & $139.4 \pm 18.5$ & $227.5 \pm 22.1$ \\
& $(n=16)^{*}$ & $(n=4)^{*}$ \\
AVNFRP & $173.1 \pm 20.0$ & $270.3 \pm 38.0$ \\
VERP & $139.5 \pm 14.3$ & $163.0 \pm 15.8$ \\
VFRP & $163.3 \pm 14.5$ & $193.3 \pm 21.3$ \\
AVNFRP - VERP $(\mathrm{ms}) \dagger$ & 33.6 & 107.3 \\
AVNFRP $>$ VERP $\ddagger$ & $21 / 21$ & $15 / 15$ \\
AVNFRP - VFRP $(\mathrm{ms}) \dagger$ & 9.8 & 77.0 \\
AVNFRP $>$ VFRP $\ddagger$ & $16 / 21$ & $15 / 15$ \\
\hline
\end{tabular}

* The remaining subjects had AVNERP atrial limited.

$\dagger$ Difference between these two parameters in ms.

$\ddagger$ Number of subjects with AVNFRP longer than VERP or VFRP.

Table 1. Resting intervals

\begin{tabular}{cccccc}
\hline & BCL $(\mathrm{ms})$ & BHR $(\mathrm{bpm})$ & PA $(\mathrm{ms})$ & AH $(\mathrm{ms})$ & HV $(\mathrm{ms})$ \\
\hline $\begin{array}{c}\text { Neonates } \\
\text { Group I } \\
(n=21)\end{array}$ & $339.9 \pm 34.1$ & $178.2 \pm 18.9$ & $7.0 \pm 5.0$ & $56.8 \pm 6.9$ & $17.8 \pm 3.1$ \\
$\begin{array}{c}\text { Adults } \\
\text { Group II } \\
(n=15)\end{array}$ & $449.5 \pm 64.6$ & $136.1 \pm 19.8$ & $12.7 \pm 9.8$ & $60.5 \pm 12.3$ & $24.5 \pm 7.8$ \\
$p$ value & $<0.001$ & $<0.001$ & $<0.05$ & NS & $<0.05$ \\
\hline
\end{tabular}


determined, the mean AVNERP was $139.4 \pm 18.5 \mathrm{~ms}$ while in the adults it measured $227.5 \pm 22.1 \mathrm{~ms}(p<0.001)$. Thus, more closely coupled supraventricular extrastimuli would be more likely to penetrate the neonatal AV node than the adult AV node.

The maximum output from the AV node is described by the AVNFRP which could be determined in all subjects. Again, the AVNFRP was significantly shorter in the neonates than in the adults (173.1 $\pm 20.0 \mathrm{~ms}$ group I; $270.3 \pm 38.0 \mathrm{~ms}$ group II, $p<$ $0.001)$.

Whether a supranodal impulse is able to reach the ventricle and induce ventricular excitation will be determined by the maximum output of the AV node (AVNFRP) and by the sensitivity or responsiveness of the ventricle (VERP) to such input. Thus, comparisons were made between the AVNFRP and VERP (Fig. 1). The AVNFRP was found to be longer than the VERP in all of the neonates $(173.1 \pm 20.0$, AVNFRP; $139.5 \pm 14.3$, VERP; $p<0.001)$ and in all adults $(270.3 \pm 38.0 \mathrm{~ms}$, AVNFRP; $163.0 \pm 21.3$, VERP; $p<0.001)$. Infranodal block $\left(\mathrm{H}_{2}\right.$ without a $V_{2}$ ) was never observed. The mean differences between these two measurements (AVNFRP and VERP) was much less in the neonates $(33.6 \mathrm{~ms})$ than in the adults $(107.3 \mathrm{~ms})$.

Previous studies concerning neonatal AV nodal function have focused their attention on the similarity of the AVNFRP and the VFRP (within $10 \mathrm{~ms}$ of each other). The mean AVNFRP exceeded the VFRP by $9.8 \mathrm{~ms}$ in the neonatal group which is similar to what has been reported previously. In our study, 16 of $21(76 \%)$ of the neonates had an AVNFRP that was longer than the VFRP. Of these, the mean AVNFRP exceeds the VFRP by $17.5 \mathrm{~ms}$. The remaining three of 21 neonatal canines had an AVNFRP equal to or shorter than the VFRP with the mean VFRP 15 ms longer than the AVNFRP. In all adults, the AVNFRP was greater than the VFRP (77 ms difference) and, again the difference between the means was larger than that observed in the neonatal group.

In $15 / 16$ of the neonates $(94 \%) \mathrm{AV}$ conduction delay during atrial extrastimulation was confined to the AVN with no change in infranodal conduction times (a type I response) (6) (Figs. 2 and 3$)$. In only $1 / 16(6 \%)$ of the neonates was there combined nodal and infranodal delay (type II response) (6). None of the neonates had increases in intranodal conduction times $\left(\mathrm{A}_{2} \mathrm{H}_{2}\right)$ of more than $25 \mathrm{~ms}$ in response to any $10 \mathrm{~ms}$ decrement in $A_{1} A_{2}$.

As previously described, the atrial extrastimulation protocol was carried out with only one extrastimulus $\left(\mathrm{S}_{2}\right)$. Despite the fact that in our neonates the AVNFRP was closer to both the VERP and VFRP than in the adults, a single atrial extrastimulation $\left(S_{2}\right)$ did not induce ventricular arrhythmias in any neonates at any

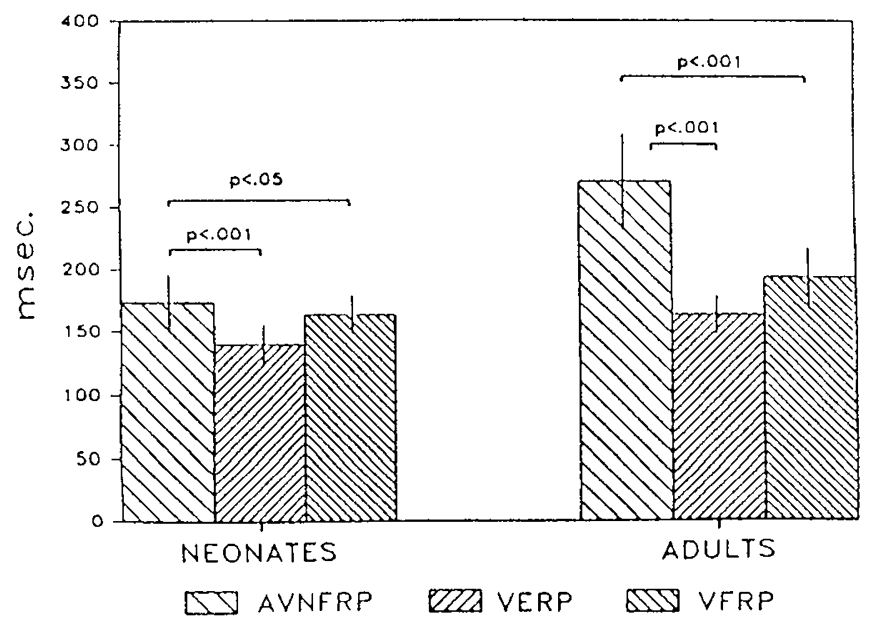

Fig. 1. Comparisons of AVNFRP, VERP, and VFRP in the neonates (group I) and adults (group II).

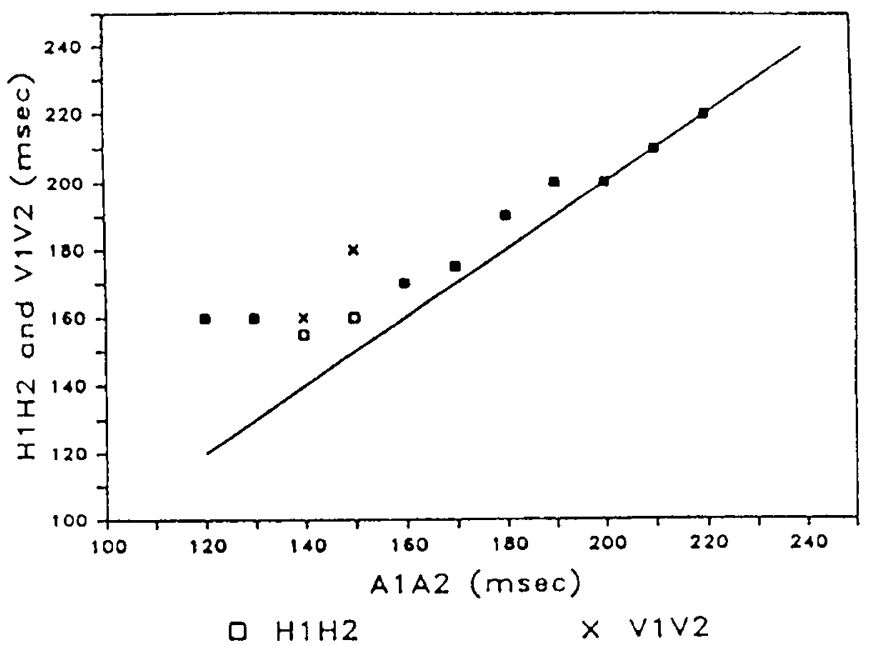

Fig. 2. Graphic representation of the response of the AV conduction system to atrial extrastimulation in a neonate. AV nodal input $\left(A_{1} A_{2}\right)$ is plotted against $\mathrm{AV}$ nodal $\left(\mathrm{H}_{1} \mathrm{H}_{2}\right)$ and $\mathrm{AV}$ conduction system $\left(\mathrm{V}_{1} \mathrm{~V}_{2}\right)$ responses. Points above the diagonal "line of identity" represent conduction delay. The shortest $\mathrm{H}_{1} \mathrm{H}_{2}$ measured (AVNFRP) is the limiting factor in antegrade conduction.

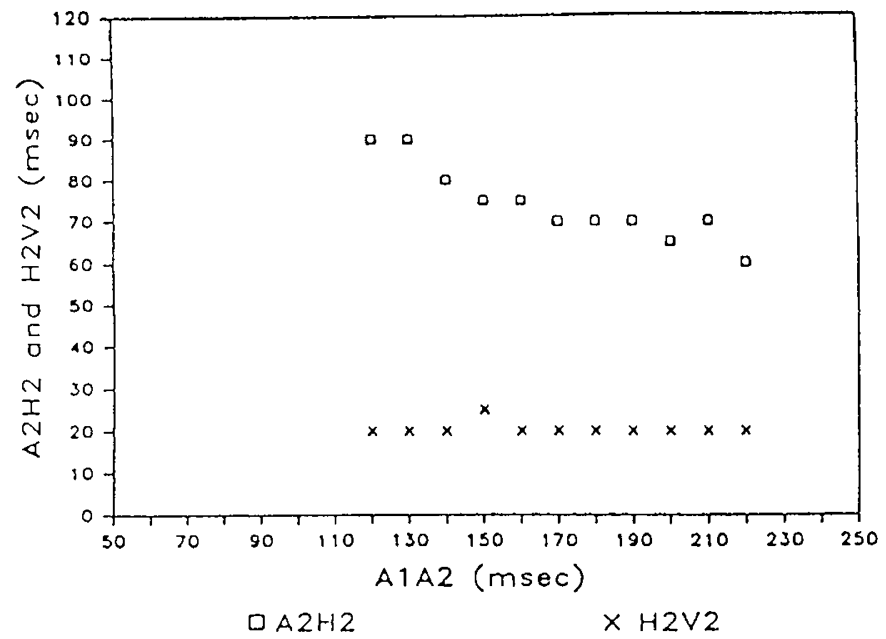

Fig. 3. Intranodal $\left(\mathrm{A}_{2} \mathrm{H}_{2}\right)$ and infranodal $\left(\mathrm{H}_{2} \mathrm{~V}_{2}\right)$ conduction intervals in response to atrial extrastimulation $\left(A_{1} A_{2}\right)$ in a neonate. At shorter $A_{1} A_{2}$ intervals, $A_{2} H_{2}$ prolongs while $H_{2} V_{2}$ remains unchanged.

$\mathrm{S}_{1} \mathrm{~S}_{2}$ interval, suggesting that in this group, the AVN renders protection to the ventricle from closely coupled supraventricular stimuli.

AV Nodal Conduction Characteristics (Antegrade and Retrograde) (Table 3). As another measure of AV nodal function, the paced heart rate at which antegrade Wenckebach periodicity occurred (WHR) was determined in both groups during incremental rapid atrial pacing. As expected, in the neonates, Wenckebach developed at a significantly faster heart rate than in the adults $(336.6 \pm 30.0 \mathrm{bpm}$ group I; $278.2 \pm 49.4 \mathrm{bpm}$ group II $p<0.001)$. However, when these results were then normalized by dividing the WHR by the BHR, similar ratios were obtained in groups I and II (Fig. 4). In the neonates, Wenckebach occurred at $1.90 \pm 0.22$ times the BHR while in the adults, Wenckebach developed at $2.05 \pm 0.20$ times the BHR. Thus, when normalized for inherent differences in resting heart rate, the neonatal and adult AVN appeared to render a similar degree of protection to the ventricle from rapid supraventricular rates.

Retrograde conduction was present in all neonates and in only $4 / 15(27 \%)$ of the adults. This is in agreement with previous 
Table 3. Wenckebach periodicity

\begin{tabular}{ccccc} 
& BHR & WHR & WHR/BHR Retro WHR* \\
\hline $\begin{array}{l}\text { Neonates } \\
\text { Group I } \\
(n=21)\end{array}$ & $178.2 \pm 18.9$ & $336.6 \pm 30.0$ & $1.90 \pm 0.22$ & $293.7 \pm 40.0$ \\
$\begin{array}{l}\text { Adults } \\
\text { Group II } \\
(n=15)\end{array}$ & $136.1 \pm 19.8$ & $278.2 \pm 49.4$ & $2.05 \pm 0.20$ & $\begin{array}{c}211.7 \pm 35.3 \\
(n=4)\end{array}$ \\
\hline
\end{tabular}

* Retro WHR, paced heart rate at which retrograde Wenckebach developed during incremental rapid ventricular pacing.
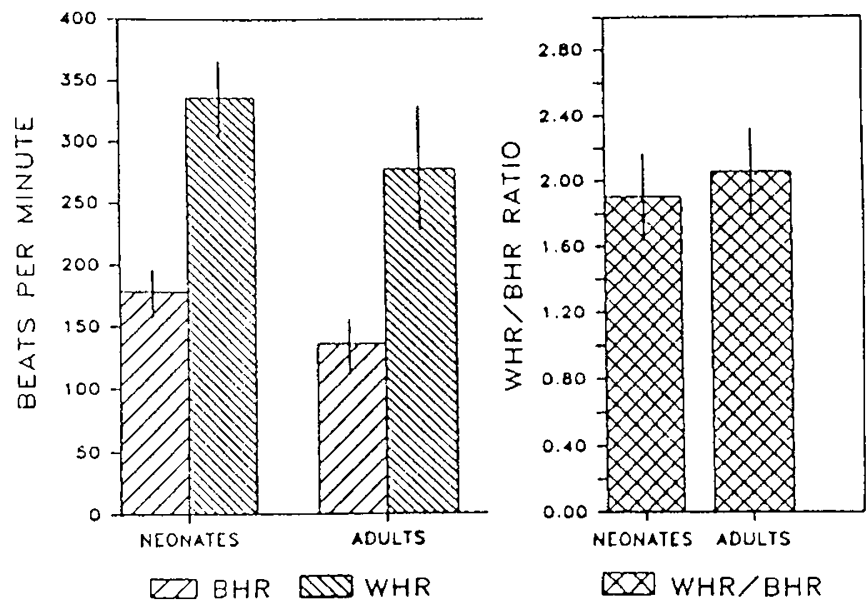

Fig. 4. Graphic comparisons of WHR, BHR, and WHR/BHR ratio in the neonates (group I) and adults (group II).

observations (5). In order to characterize the functional characteristics of the retrograde conduction system, a comparison was made between the heart rates at which retrograde and antegrade Wenckebach occurred. In the neonates, antegrade Wenckebach occurred at $336.6 \pm 30.0 \mathrm{bpm}$ while retrograde Wenckebach occurred at $293.7 \pm 40.0 \mathrm{bpm}(p<0.001)$. Thus, while retrograde conduction is uniformly present in the neonates, it appears that antegrade conduction is somewhat better than retrograde conduction, with Wenckebach occurring at faster heart rates in the antegrade direction.

\section{DISCUSSION}

Very few studies concerning the functional properties of the immature AV node and AV conduction system have been reported. Preston et al. (1) studied the AV conduction system in open chest pentobarbital anesthetized young goats, pigs, and puppies. These investigators reported that arrhythmias were commonly induced by atrial extrastimulation in the young goats and pigs (but not in 6- to 12-wk-old puppies). In that study, AV nodal conduction characteristics were inferred (no recording of His bundle electrograms) and one of the major premises of this study was that the ventriculoatrial (retrograde) refractory period was equivalent to the antegrade AV nodal refractoriness. They concluded that the AVNFRP is shorter than the VFRP and that limitation to AV conduction in the immature heart is imposed by the ventricle itself.

Moore (2) studied open chest pentothal anesthetized calves. Although in that study no ventricular arrhythmias were inducible by atrial pacing, there were no increases observed in AVN conduction time in response to prematurely delivered extrastimuli. Instead, AV conduction delay was confined to the His Purkinje system. The conclusion of this study was, again, that in the immature heart the AVNFRP is shorter than the VFRP.

Gough and Moore (3) studied AV conduction in open chest pentobarbital anesthetized goats. They concluded that in the neonatal goat, the AVNFRP falls within $10 \mathrm{~ms}$ of the VFRP and, that because of this, ventricular arrhythmias were commonly induced during atrial extrastimulation. This functional immaturity of the neonatal AV conduction system has been implicated as a possible cause of sudden death in infancy (4).

In our model we utilized $\mathrm{Na}$ pentobarbital as the anesthetic agent. Other studies have suggested that $\mathrm{Na}$ pentobarbital may exert a vagolytic effect, which would tend to shorten AV nodal refractoriness. Despite this, our results show that AV nodal refractoriness is longer than that of the ventricle.

In our study, we present evidence that suggests that at least in the neonatal canine, the AVN does render protection to the ventricles from closely coupled atrial extrastimuli and rapid supraventricular rates. Contrary to the previously cited reports, we have shown that in the neonatal canine, the mean AVNFRP is, in the majority of subjects, longer than the VFRP. Furthermore, ventricular arrhythmias were not induced by a single atrial extrastimulation. However, $\mathrm{S}_{3}$ and $\mathrm{S}_{4}$ were not employed, which may or may not have resulted in ventricular arrhythmias. In our neonates, it was observed that the AVNFRP was always longer than the VERP. Block distal to the bundle of His during atrial extrastimulation never occurred in the neonates, again, in contrast to the previously cited reports. In our study, the neonatal AV node acted as the primary site of conduction delay in the $\mathrm{AV}$ conduction system and, as a rule, was the limiting site of conduction during extrastimulation. Furthermore, while the resting supraventricular rate is faster in the neonate, the $\mathrm{AH}$ interval was the same in both groups, suggesting that the neonates have a relatively longer AV nodal conduction time. Thus, our data provide evidence that the neonatal AV nodal conduction characteristics resemble those of the adult more so than previous described.

Our findings concerning the development of Wenckebach periodicity in the immature canine heart provides further support for the concept that the neonatal AV node is capable of protecting the ventricles from relatively rapid supraventricular rates. Although the neonatal AV node developed Wenckebach periodicity at a faster heart rate than in the adult, when normalized for the resting heart rates in both groups, Wenckebach developed at a heart rate of approximately twice the resting heart rate in each group. While we recognize that the present experimental conditions do not necessarily replicate the conditions during naturally occurring supraventricular dysrhythmias (when the AV node may be subjected to increased adrenergic stimulation and may exhibit enhanced conduction characteristics) at least in the controlled setting of the laboratory, under identical conditions, and during identical pacing protocols, we find little or no differences between the neonatal and adult AV node in the relative degree of protection offered against rapid supraventricular rates as assessed by the development of Wenckebach.

While our findings concerning the AVNFRP-VFRP are in agreement with those of Gough and Moore (3) in that there is a 10 -ms difference between these two values, the rest of our findings lead us to arrive at different conclusions. In their work, Gough and Moore (3) did not evaluate the AVNFRP-VERP relationship, which indeed is what will determine whether or not a supraventricular impulse will reach the ventricle. We found this difference to be $33 \mathrm{~ms}$ and this seems to be large enough to prevent supraventricular impulses from reaching the ventricle in its vulnerable period, as evidenced by the fact that ventricular arrhythmias induced by atrial extrastimulation were never observed. Our findings regarding the Wenckebach periodicity also support our conclusion that the neonatal AV node does render adequate protection to the ventricle from rapid supraventricular rates.

The apparent differences in the results from our study and those of previous investigations may be due in part to species specific differences and differences of age even within the same species. Our neonatal puppies were 3-15 days old while Preston et al. (1) studied puppies significantly older (6-12 wk old). 
Furthermore, while most of the previous work had been done utilizing open chest preparations, we have used a less invasive closed chest model which may account for the differences observed. It is possible, for example, that the open chest preparation results in an increased level of sympathetic activity which could shift AV nodal function in the direction of enhanced conduction, although previous studies (3) utilized pharmacologic autonomic blockade. Another finding in our study was that in the neonates, AV nodal delay was the rule during atrial extrastimulation rather than infranodal delay as reported by others. Again, differences in experimental design, autonomic state, and species-specific characteristics probably account for these differences.

In summary, contrary to previous reports concerning neonatal AV nodal function, our data suggest that in the neonatal canine, the AV node is more functionally mature than previously described. It renders adequate protection to the ventricles against premature and rapid supraventricular rates by virtue of its longer refractoriness and antegrade delay characteristics. Thus, generalizations concerning normal neonatal AV nodal function in the human must be tempered by an appreciation that information derived from animal studies may or may not be directly appli- cable to the human. Important species- and age-specific differences exist in AV nodal function. The functional maturity of the human neonatal AVN can only be definitively assessed by electrophysiologic studies in the human.

Acknowledgment. The authors thank Diane McMullen for her excellent secretarial work.

\section{REFERENCES}

1. Preston JB, McFadden S, Moe GK 1959 Atrioventricular transmission in young mammals. Am J Physiol 197:236-240

2. Moore EN 1965 Atrioventricular transmission in newborn calves. Ann NY Acad Sci 127:113-126

3. Gough WB, Moore EN 1975 the differences in atrioventricular conduction of premature beats in young and adult goats. Circ Res 37:48-58

4. Dawes GS 1968 Sudden death in babies: physiology of the fetus and newborn. Am J Cardiol 22:469-478

5. Pickoff AS, Singh S, Flinn CJ, Torres E, Ezrin A, Gelband H 1984 Maturational changes in ventriculoatrial conduction in the inta $t$ canine heart. $\mathbf{J}$ Am Coll Cardiol 3:162-168

6. Josephson ME, Seides SF 1979 Electrophysiologic Investigation: General Concepts, In: Josephson ME, Seides SF (eds) Clinical Cardiac Electrophysiology, Techniques and Interpretations. Lea \& Febiger, Philadelphia, pp 23-59 